\title{
APPROXIMATE CALCULATION OF THE NATURAL OSCILLATION FREQUENCY OF THE VIBRATING TABLE IN INTER-RESONANCE OPERATION MODE
}

\author{
MAISTRUK Pavlo ${ }^{1 *}$, LANETS Oleksii ${ }^{1}$, STUPNYTSKYY Vadym ${ }^{1}$ \\ ${ }^{I}$ Department of Robotics and Integrated Mechanical Engineering Technologies, Lviv Polytechnic \\ National University, Institute of Engineering Mechanics and Transport, Profesorska 1, Lviv, Ukraine, \\ e-mail: pmaystruk@gmail.com
}

\begin{abstract}
The first natural frequency of oscillations of the vibrations of a discrete-continuous interresonant vibrating table plate was found using the functions of the Krylov-Duncan. The continuous member, presented in a plate hinged at four points, is used as a reactive mass with distributed parameters to create an inter-resonance vibrating table with an electromagnetic drive. For this purpose, the plate was considered as a rod on hinged supports. The justification of this approach is confirmed by simulation in the Ansys software. The results were confirmed using the approximate Rayleigh-Ritz method. The geometric shape of the plate is reduced to a section of a hyperboloidal surface. The results of simulation of the first natural frequency were verified experimentally.
\end{abstract}

KEYWORDS: inter-resonance vibrating table, continuous member, elastic plate, the natural frequency of body oscillations, Rayleigh-Ritz method.

\section{Introduction}

Nowadays, vibration technologies are used in many industries (mechanical engineering, civil engineering, food industry, and others). One of the common types of vibrating technological equipment is vibrating tables. They are designed for vibro-compaction of viscous mixtures and bulk materials when minimal porosity is required.

Modern trends in industrial development require energy-saving equipment, which reduces the cost of manufacturing the product. To date, one- and two-mass vibrating machines have become widespread [1]. Due to the simplicity of manufacture and operation, they are efficient and relatively cheap for vibrating technological equipment. Such equipment satisfies almost all production needs. There are also three-mass vibrating machines with much better energy efficiency through inter-resonance modes of operation [2]. Such machines are a promising area of research, although they have more complex design.

The paper [2] extensively describes the methodology for creating three-mass interresonant vibrating machines. Combining the two resonant peaks allows the oscillating system to accumulate a high dynamic potential in a relatively narrow frequency range. Because of this, such oscillatory systems can have much higher mass dynamics coefficients than two-mass resonance systems and are therefore considered more highly efficient. Energy efficiency is achieved because much higher amplitudes of mass oscillations can be provided for the same values of disturbing forces.

A significant disadvantage of highly efficient three-mass oscillatory systems is that the third reactive mass is small, and its amplitude in the inter-resonance zone increases rapidly [3]. This complicates the provision of inter-resonance mode, especially in systems with electromagnetic 
drive (beating the armature to the iron core of the electromagnetic vibrator). The unbalanced drive is not able to enter the inter-resonance zone due to the presence of the Sommerfeld effect.

In highly efficient designs of inter-resonance vibrating machines, there is a noticeable tendency to reduce the inertial value of the reactive mass, which allows them to use energysaving inter-resonance modes of operation effectively. It indicates that the reactive mass and stiffness of the corresponding elastic node of the inter-resonance vibratory machines must be ultra-small. It is possible only by using a flexible body, i.e. a continuous member, which optimally combines inertial and rigid parameters. The authors made this decision in the article [4]. However, the disadvantage of the design considered in the article is that the perturbation of the continuous member in the form of a rod occurs from the crank drive. This involves using a controlled mechanism that ensures the smooth entry of the oscillating system into the interresonance zone. In addition, a significant disadvantage of such perturbation is that there is intense destruction of the crank mechanism containing precision moving joints.

The disadvantages mentioned above are absent in vibrating machines with an electromagnetic drive. However, in three-mass inter-resonance systems, it is rarely used due to a rapid amplitude-frequency characteristic of the reactive mass, which leads to the beating of the armature to the iron core of the electromagnets. Given the prospects for developing interresonance vibratory machines with continuous members and the significant advantages of electromagnetic drive, this article aims to develop approaches in creating discrete-continuous inter-resonance vibrating tables with electromagnetic drive. Using as a continuous member a plate, perturbed by the electromagnetic drive, is optimal. The plate has a significant surface that can overlap the poles of the electromagnetic vibrator.

The fundamental frequency is the first natural frequency of the line, and it usually has the vibration peak with the highest value. An elastic plate has many natural frequencies and mode shapes, where each mode shape corresponds to a particular frequency value. Therefore, the frequency analysis of elastic plates is relevant.

The articles [5 - 10] carried out the vibration analysis of functionally graduated (FG) plates and beam structures. The primary attention is paid to determining the properties of the plates when changing their materials. Different boundary conditions for fixing FG plates (boundary conditions of the types SSSS or CCCC) are also considered. More complex boundary conditions for fixing elastic plates (boundary conditions of the types CCSS, CSSS, CFSS, SFSS, SSSF, etc.) are solved using the Levy solution [11].

A significant contribution to the development of methods for calculating elastic plates and shells was made by S. Tymoshenko [12]. It was shown in [13] that the classical structure of the potential energy of a deformed nanoplate is preserved with the redefined elastic moduli, which contain the characteristics of a body and a surface. Mahi A. et al. [14] presented a new hyperbolic shear deformation theory, which can apply to bending and free vibration analysis of isotropic, functionally graded, sandwich, and laminated composite plates. Vescovini R. et al. [15] present a new vision of using the Ritz method for free vibration and buckling analysis of composite plates. The main attention is paid to the choice of the trial functions concerning the degree and the kind of anisotropy exhibited by the plates. In [16], accepting Kirchhoff's hypotheses about the normality of elements concerning the median surface before and after loading, a method for calculating the ultimate true stresses arising in the walls of shells of revolution in the area of uniform plastic deformation is proposed. Arvin H. [17] analyzed the free oscillations of Tymoshenko's microbeams using the differential transform method. There is also the possibility of using a variational method of iterations to analyze Tymoshenko's beams' free oscillations [18]. The article [19] presents numerical and experimental analysis of the state of stress in a circular perforated plate, using Ansys software. Hlavaty M. et al. [20] 
describe characteristic properties of quasi-Rayleigh waves that are important for ultrasonic defect detection of structural plates.

Therefore, in this article, the authors will try to establish the natural frequency of the continuous member in the form of a plate with complex boundary conditions, which will be used in a discrete-continuous inter-resonance vibrating table with an electromagnetic drive.

\section{Description of the problem}

The authors developed a discrete-continuous inter-resonance vibrating table with an electromagnetic drive, the schematic diagram shown in Fig. 1. This vibrating machine consists of active, intermediate, and reactive masses. The active and intermediate masses are discrete (we consider them to be solid bodies), and the reactive mass is continuous (we consider it a body with distributed parameters). These oscillating masses are connected in series through elastic units. Note that the reactive mass, as a body with distributed parameters, combines inertial and rigid parameters. These parameters appear only in dynamic processes as abstract distributed values. The oscillation masses of the vibratory machine are denoted as $m_{1}, m_{2}, m_{3}$ , and the rigidities of the elastic units - as $c_{12}$ and $c_{23}$. The active mass is attached to the foundation through vibration isolators with rigidity $c_{i s}$.

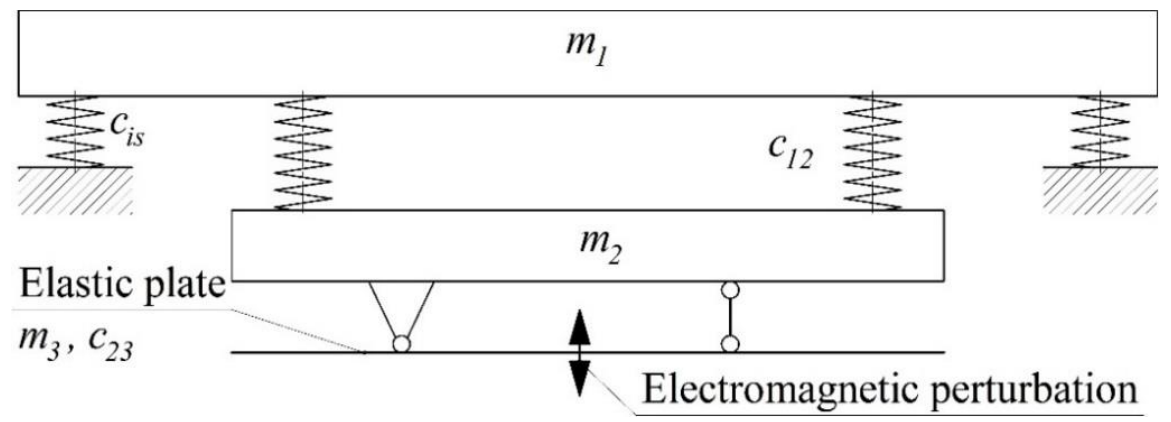

Fig. 1 Scheme of the inter-resonance vibrating table

This inter-resonance vibrating table's reactive mass (continuous member) should be light and have the necessary inertial-rigid parameters. Therefore, as a reactive mass, it is advisable to use an elastic plate. The elastic plate is fixed in the intermediate-mass by a hinged connection (Fig. 2).

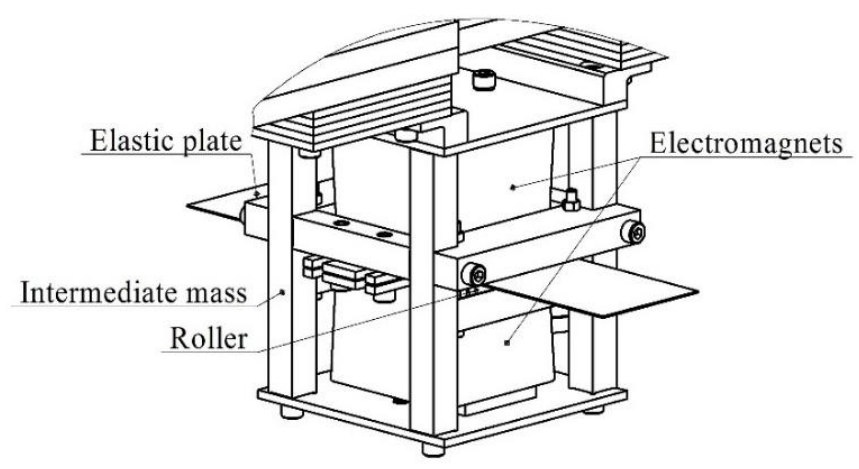

Fig. 2 Fastening of a continuous member in a vibrating table

As is known [2], the parameters of rigidity and mass of the plate are related to the parameter of its natural oscillation frequency by the equation: 


$$
\omega=\sqrt{\frac{c_{23}}{m_{3}}}
$$

Therefore, the essential parameter in the synthesis of a discrete-continuous system is the natural frequency of the plate. After all, the proper selection of the natural frequency of the reactive mass allows us to obtain a highly efficient mode of operation of discrete-time interresonant vibrating machines.

As a plate material, we choose structural steel. This material is a ferromagnet and has a relatively low cost. The scheme of fastening of an elastic plate is shown in Fig. 3.

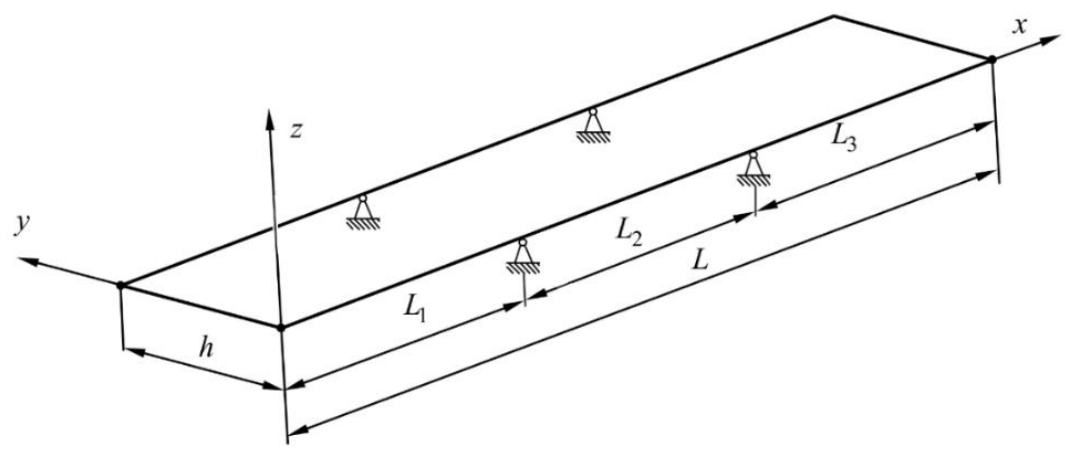

Fig. 3 Calculation scheme of elastic plate mounting

As shown in Fig. 3, the fastening of the elastic plate does not allow obtaining an exact solution using known calculation methods [11]. Therefore, the task of this article is to establish the first natural oscillation frequency of the plate with this type of fastening using approximate calculation methods.

\section{Calculation of the natural frequency of the plate using Krylov-Duncan functions}

In this paper, we assume that the length of the plate is much larger than the width $(L>>h)$, and therefore the plate can be considered a rod that performs only bending oscillations in the plane $0 x z$ (Fig. 3). The assumption that the plate is a rod simplifies the calculations without making a significant error. This statement will be proved below. Therefore, to describe the calculation of natural frequency, the authors will use the term "rod" in the future. We use the classical theory of oscillations of straight elastic rods [12] and Krylov-Duncan functions [21].

The proposed calculation scheme for fastening the rod is shown in Fig. 4. The hinged mounting of the rod allows us to get a console with significant inertia, which is driven by electromagnets. It is assumed that in this case, the conditionally allocated mass and rigidity will be perceived by the oscillating system as an independent mass, and the discrete-continuous oscillating system will be inter-resonance.

For further calculations, the rod is divided into three sections. They are interconnected through reactions $R_{1}$ and $R_{2}$ in hinged supports. Denote the ratio of the distances of the sections to the total length of the rod:

$$
\begin{gathered}
L_{1} / L=L_{3} / L=\beta, \\
L_{2} / L=\gamma .
\end{gathered}
$$

The deflection of any section can be given as an equation [21]:

$$
w(x)=A \cdot S(\xi x)+B \cdot T(\xi x)+C \cdot U(\xi x)+D \cdot V(\xi x),
$$


where $A, B, C, D$ - coefficients, determined through boundary conditions;

$S(\xi x), T(\xi x), U(\xi x)$ and $V(\xi x)$ are Krylov-Duncan functions:

$S(\xi x)=(\operatorname{ch}(\xi x)+\cos (\xi x)) / 2 ; T(\xi x)=(\operatorname{sh}(\xi x)+\sin (\xi x)) / 2 ;$

$U(\xi x)=(\operatorname{ch}(\xi x)-\cos (\xi x)) / 2 ; V(\xi x)=(\operatorname{sh}(\xi x)-\sin (\xi x)) / 2$.

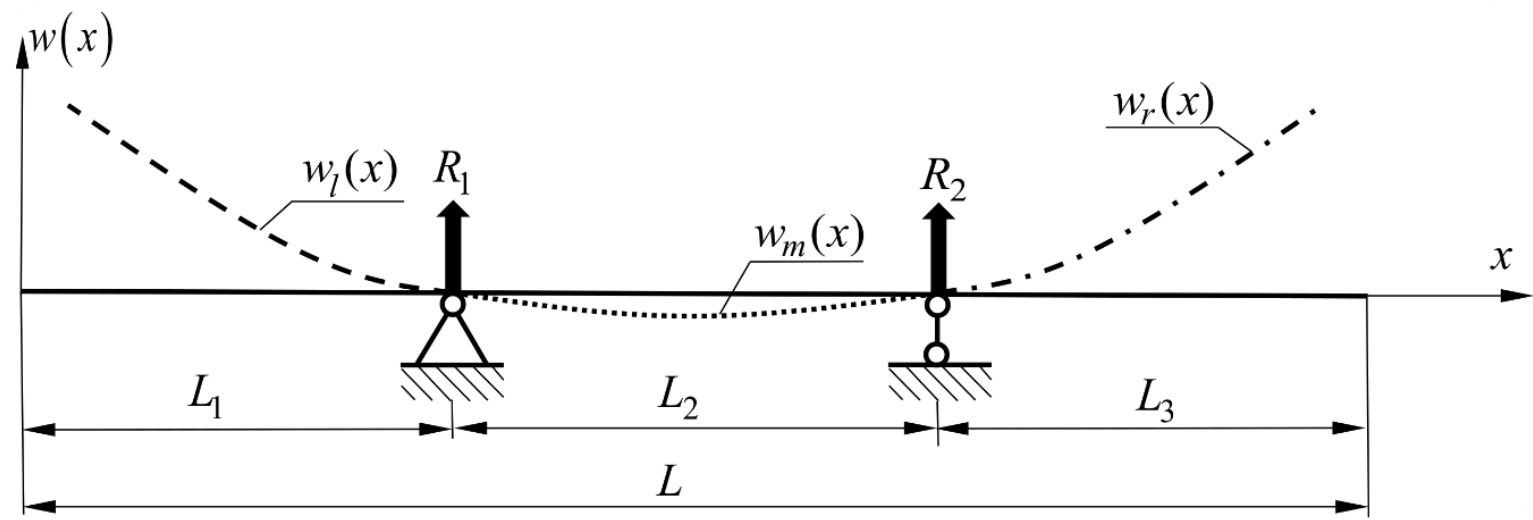

Fig. 4 The calculated scheme of the rod with distributed parameters, where $L_{1}, L_{3}$ - distances from the left and right end according to the left and right hinged supports; $L_{2}$ - distances between hinged supports; $L$ - the length of the rod; $R_{1}, R_{2}$ - reactions in the hinged supports

The coefficient $\xi$ can be found from the following equation:

$$
\xi=\sqrt[4]{\frac{m_{r m} \omega^{2}}{E J_{z}}},
$$

where $m_{r m}$ - the mass of the running meter of the rod; $\omega$ - circular frequency of harmonic oscillations of the rod; $E=2,1 \cdot 10^{11} \mathrm{~Pa}$ - Young's modulus for steel; $J_{z}$ - moment of inertia of the rectangular cross section of the rod relative to the neutral line.

The equation of deflections of the left $w_{l}(x)$, middle $w_{m}(x)$ and right $w_{r}(x)$ sections of the rod, based on Fig. 4, we will connect according to dependences [21]:

$$
\begin{gathered}
w_{m}(x)=w_{l}(x)+\frac{R_{1}}{\xi^{3} E J_{z}} \cdot V_{2}(\xi L(1-\beta)) ; \\
w_{r}(x)=w_{m}(x)+\frac{R_{2}}{\xi^{3} E J_{z}} \cdot V_{3}(\xi L(1-\beta-\gamma))= \\
=w_{l}(x)+\frac{R_{1}}{\xi^{3} E J_{z}} \cdot V_{2}(\xi L(1-\beta))+\frac{R_{2}}{\xi^{3} E J_{z}} \cdot V_{3}(\xi L(1-\beta-\gamma)),
\end{gathered}
$$

where $V_{2}(\xi L(1-\beta))=(\operatorname{sh}(\xi L(1-\beta))-\sin (\xi L(1-\beta))) / 2$;

$V_{3}(\xi L(1-\beta-\gamma))=(\operatorname{sh}(\xi L(1-\beta-\gamma))-\sin (\xi L(1-\beta-\gamma))) / 2-$ corresponding KrylovDuncan functions. 
Let's take the left end of the rod, which is in the free state, as the starting point. In this case, there is no bending moment and shear force. Following this, we make equations of deflections of the left section. For these conditions, we assume that the initial conditions at this end will be:

$$
\begin{aligned}
& \left.\frac{\mathrm{d}^{2} w_{l}(x)}{\mathrm{d} x^{2}}\right|_{x=0}=\xi^{2} C=0, \\
& \left.\frac{\mathrm{d}^{3} w_{l}(x)}{\mathrm{d} x^{3}}\right|_{x=0}=\xi^{3} D=0 .
\end{aligned}
$$

Then the general equation in the left section $\left(0 \leq x \leq L_{1}\right)$, substituting the initial conditions (7) and (8) in expression (4), is:

$$
w_{l}(x)=A \cdot S(\xi x)+B \cdot T(\xi x) .
$$

The equation of deflections $w_{m}$ on the middle section of the $\operatorname{rod}\left(L_{1} \leq x \leq L_{1}+L_{2}\right)$, substituting (9) in the first expression (6), will take the form:

$$
w_{m}(x)=A \cdot S(\xi x)+B \cdot T(\xi x)+\frac{R_{1}}{\xi^{3} E J_{z}} \cdot V_{2}(\xi L(1-\beta)) .
$$

In the right section $\left(L_{1}+L_{2} \leq x \leq L\right)$, substituting (9) in the second expression (6), the equation of deflections will look like:

$$
w_{r}(x)=A \cdot S(\xi x)+B \cdot T(\xi x)+\frac{R_{1}}{\xi^{3} E J_{z}} \cdot V_{2}(\xi L(1-\beta))+\frac{R_{2}}{\xi^{3} E J_{z}} \cdot V_{3}(\xi L(1-\beta-\gamma)) .
$$

Equations (9) - (11) are written in general form. To determine the first natural frequency of the rod, it is necessary to compose a system of equations that will satisfy the boundary conditions at the right end. The right end of the rod along the length (Fig. 4) is free, so the bending moment and shear force on it, which are the second and third derivatives of the deflection function, are zero. Thus, the initial conditions of the rod fastening will be:

$$
\begin{aligned}
& \left.\frac{\mathrm{d}^{2} w_{r}(x)}{\mathrm{d} x^{2}}\right|_{x=L}=0, \\
& \left.\frac{\mathrm{d}^{3} w_{r}(x)}{\mathrm{d} x^{3}}\right|_{x=L}=0 .
\end{aligned}
$$

The first equation is formed according to condition (12). The second derivative of the deflection of the end of the right section, which symbolizes momentum, is zero. Therefore, by differentiating equation (11) twice, we obtain:

$$
\begin{gathered}
\left.\frac{\mathrm{d}^{2} w_{r}(x)}{\mathrm{d} x^{2}}\right|_{x=L}=A \xi^{2} \cdot U_{1}(\xi L)+B \xi^{2} \cdot V_{1}(\xi L)+\frac{R_{1} \xi^{2}}{\xi^{3} E J_{z}} \times \\
\times T_{2}(\xi L(1-\beta))+\frac{R_{2} \xi^{2}}{\xi^{3} E J_{z}} \cdot T_{3}(\xi L(1-\beta-\gamma)),
\end{gathered}
$$

where $U_{1}(\xi L)=(\operatorname{ch}(\xi L)-\cos (\xi L)) / 2$;

$V_{1}(\xi L)=(\operatorname{sh}(\xi L)-\sin (\xi L)) / 2$; 


$$
\begin{aligned}
& T_{2}(\xi L(1-\beta))=(\operatorname{sh}(\xi L(1-\beta))+\sin (\xi L(1-\beta))) / 2 \\
& T_{3}(\xi L(1-\beta-\gamma))=(\operatorname{sh}(\xi L(1-\beta-\gamma))+\sin (\xi L(1-\beta-\gamma))) / 2 .
\end{aligned}
$$

The second equation is formed according to condition (13), where the third derivative of the deflection of the end of the right section is equal to zero. By differentiating equation (11) three times and substituting the value $x=L$, we obtain:

$$
\begin{gathered}
\left.\frac{\mathrm{d}^{3} w_{r}(x)}{\mathrm{d} x^{3}}\right|_{x=L}=A \xi^{3} \cdot T_{1}(\xi L)+B \xi^{3} \cdot U_{1}(\xi L)+ \\
+\frac{R_{1} \xi^{3}}{\xi^{3} E J_{z}} \cdot S_{2}(\xi L(1-\beta))+\frac{R_{2} \xi^{3}}{\xi^{3} E J_{z}} \cdot S_{3}(\xi L(1-\beta-\gamma)),
\end{gathered}
$$

where $T_{1}(\xi L)=(\operatorname{sh}(\xi L)+\sin (\xi L)) / 2$;

$S_{2}(\xi L(1-\beta))=(\operatorname{ch}(\xi L(1-\beta))+\cos (\xi L(1-\beta))) / 2$;

$S_{3}(\xi L(1-\beta-\gamma))=(\operatorname{ch}(\xi L(1-\beta-\gamma))+\cos (\xi L(1-\beta-\gamma))) / 2$.

Since we have four unknowns: constants $A, B$ and reactions on hinged supports $R_{1}$ and $R_{2}$, we operate with only two equations (14) and (15), we need to add two more equations. We will form them from the condition of zero deflection on supports. At length $x=L_{1}=L \cdot \beta$, using the deflection equation of the left section (9), the equation will look like:

$$
w_{l}(\xi x)_{x=L \cdot \beta}=A \cdot S_{4}(\xi L \cdot \beta)+B \cdot T_{4}(\xi L \cdot \beta)=0,
$$

where $S_{4}(\xi L \cdot \beta)=(\operatorname{ch}(\xi L \cdot \beta)+\cos (\xi L \cdot \beta)) / 2$;

$T_{4}(\xi L \cdot \beta)=(\operatorname{sh}(\xi L \cdot \beta)+\sin (\xi L \cdot \beta)) / 2$.

For the middle section of the plate, where $x=L_{1}+L_{2}=L(\beta+\gamma)$, using equation (10):

$$
w_{m}(\xi x)_{x=L(\beta+\gamma)}=A \cdot S_{5}(\xi L(\beta+\gamma))+B \cdot T_{5}(\xi L(\beta+\gamma))+\frac{R_{1}}{\xi^{3} E J_{z}} \cdot V_{5}(\xi L \cdot \gamma)=0 \text {, }
$$

where $S_{5}(\xi L(\beta+\gamma))=(\operatorname{ch}(\xi L(\beta+\gamma))+\cos (\xi L(\beta+\gamma))) / 2$;

$T_{5}(\xi L(\beta+\gamma))=(\operatorname{sh}(\xi L(\beta+\gamma))+\sin (\xi L(\beta+\gamma))) / 2 ;$

$V_{5}(\xi L \cdot \gamma)=(\operatorname{sh}(\xi L \cdot \gamma)-\sin (\xi L \cdot \gamma)) / 2$.

Therefore, taking into account (14) - (17), we obtain a system of four equations that describes the natural oscillations of the rod: 


$$
\left\{\begin{array}{c}
A \xi^{2} U_{1}(\xi L)+B \xi^{2} V_{1}(\xi L)+\frac{R_{1}}{\xi E J_{z}} \cdot T_{2}(\xi L(1-\beta))+\frac{R_{2}}{\xi E J_{z}} \cdot T_{3}(\xi L(1-\beta-\gamma))=0 \\
A \xi^{3} T_{1}(\xi L)+B \xi^{3} U_{1}(\xi L)+\frac{R_{1}}{E J_{z}} \cdot S_{2}(\xi L(1-\beta))+\frac{R_{2}}{E J_{z}} \cdot S_{3}(\xi L(1-\beta-\gamma))=0 \\
A \cdot S_{4}(\xi L \cdot \beta)+B \cdot T_{4}(\xi L \cdot \beta)=0 \\
A \cdot S_{5}(\xi L(\beta+\gamma))+B \cdot T_{5}(\xi L(\beta+\gamma))+\frac{R_{1}}{\xi^{3} E J_{z}} \cdot V_{5}(\xi L \cdot \gamma)=0 .
\end{array}\right.
$$

From the system of equations (18), we highlight the frequency matrix:

$$
\Delta(\xi L)=\left|\begin{array}{cccc}
U_{1}(\xi L) & V_{1}(\xi L) & T_{2}(\xi L(1-\beta)) & T_{3}(\xi L(1-\beta-\gamma)) \\
T_{1}(\xi L) & U_{1}(\xi L) & S_{2}(\xi L(1-\beta)) & S_{3}(\xi L(1-\beta-\gamma)) \\
S_{4}(\xi L \cdot \beta) & T_{4}(\xi L \cdot \beta) & 0 & 0 \\
S_{5}(\xi L(\beta+\gamma)) & T_{5}(\xi L(\beta+\gamma)) & V_{5}(\xi L \cdot \gamma) & 0
\end{array}\right|,
$$

whose determinant is written as:

$$
\begin{gathered}
\Delta(\xi L)=U_{1}(\xi L) \cdot T_{4}(\xi L \cdot \beta) \cdot S_{3}(\xi L(1-\beta-\gamma)) \cdot V_{5}(\xi L \cdot \gamma)-T_{1}(\xi L) \cdot T_{4}(\xi L \cdot \beta) \times \\
\times T_{3}(\xi L(1-\beta-\gamma)) \cdot V_{5}(\xi L \cdot \gamma)-S_{4}(\xi L \cdot \beta) \cdot V_{1}(\xi L) \cdot S_{3}(\xi L(1-\beta-\gamma)) \cdot V_{5}(\xi L \cdot \gamma)+ \\
+S_{4}(\xi L \cdot \beta) \cdot U_{1}(\xi L) \cdot T_{3}(\xi L(1-\beta-\gamma)) \cdot V_{5}(\xi L \cdot \gamma)+S_{4}(\xi L \cdot \beta) \cdot T_{5}(\xi L(\beta+\gamma)) \times \\
\times T_{2}(\xi L(1-\beta)) \cdot S_{3}(\xi L(1-\beta-\gamma))-S_{4}(\xi L \cdot \beta) \cdot T_{5}(\xi L(\beta+\gamma)) \cdot T_{3}(\xi L(1-\beta-\gamma)) \times \\
\quad \times S_{2}(\xi L(1-\beta))-S_{5}(\xi L(\beta+\gamma)) \cdot T_{4}(\xi L \cdot \beta) \cdot T_{2}(\xi L(1-\beta)) \cdot S_{3}(\xi L(1-\beta-\gamma))+ \\
\quad+S_{5}(\xi L(\beta+\gamma)) \cdot T_{4}(\xi L \cdot \beta) \cdot T_{3}(\xi L(1-\beta-\gamma)) \cdot S_{2}(\xi L(1-\beta)) .
\end{gathered}
$$

To find the value of the determinant of the frequency matrix, set the length of the sections of the rod: $L=0,54 m, L_{1}=0,182 m, L_{2}=0,176 m, L_{3}=0,182 \mathrm{~m}$. Using the software product MathCAD, we will graphically display the results of the calculation of the determinant of the frequency matrix on the interval $\xi L \in[0 \ldots 6]$ (Fig. 5). The first zero value of the determinant (20), symbolizing the first natural frequency, is observed at $\xi L=4,2146$. 


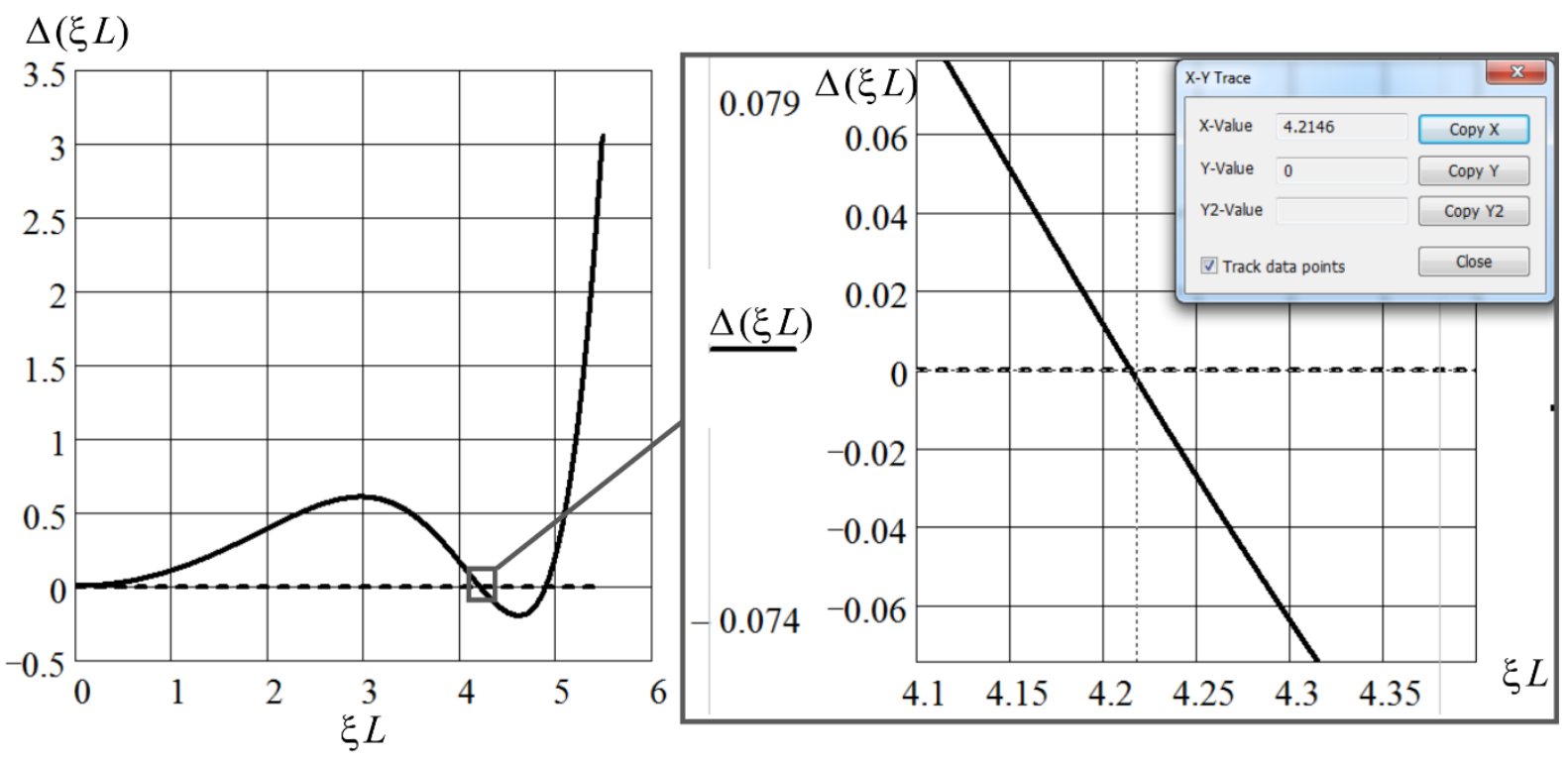

Fig. 5 MathCAD software window, which graphically displays the solution of the frequency matrix determinant

Before setting the circular frequency $\omega$ of the natural oscillations of the rod, determine the mass $m_{r m}$ of its running meter. We assume the cross-section of the elastic rod is rectangular so that the equation can be written as:

$$
m_{r m}=\rho h b_{n}=2,335 \mathrm{~kg} / \mathrm{m},
$$

where $\rho=7850 \mathrm{~kg} / \mathrm{m}^{3}-$ density of steel;

$h=0,0875 m-$ width of the rod;

$b_{n}=3,4 \cdot 10^{-3} m-$ thickness of the rod.

The moment of inertia of a rectangular cross-section of the rod concerning the neutral line is determined according to:

$$
J_{z}=\frac{h b_{n}^{3}}{12}=2,866 \cdot 10^{-10} \mathrm{~m}^{4} .
$$

Therefore, the natural frequency of oscillations of the rod as a body with distributed parameters using formula (5) will be:

$$
\omega=(\xi L)^{2} \sqrt{\frac{E J_{z}}{m_{r m} L^{4}}}=309,24 \mathrm{rad} / \mathrm{s} .
$$

Accordingly, the cyclic frequency of natural oscillations of the rod will be:

$$
v=\frac{\omega}{2 \pi}=49,22 \mathrm{~Hz}
$$

Thus, considering the plate as a rod, its first natural frequency of oscillations is established using Krylov-Duncan functions. The reliability of the presented calculation of natural frequency will be checked in the Ansys software. 


\section{Calculation of the natural frequency of the plate using the finite element method (FEM) in the Ansys software}

This part of the paper is devoted to numerical calculation methods using the Modal software module, which runs in the Ansys shell and aims at confirming the results obtained in item 3. This will indirectly confirm the adequacy of both the calculation methodology and the given system parameters.

A solid plate model with all the parameters, such as geometric dimensions, material, and type of fastening, was exported to the Ansys software. The result of modelling this plate in the software product is shown in Fig. 7 and Fig. 8.

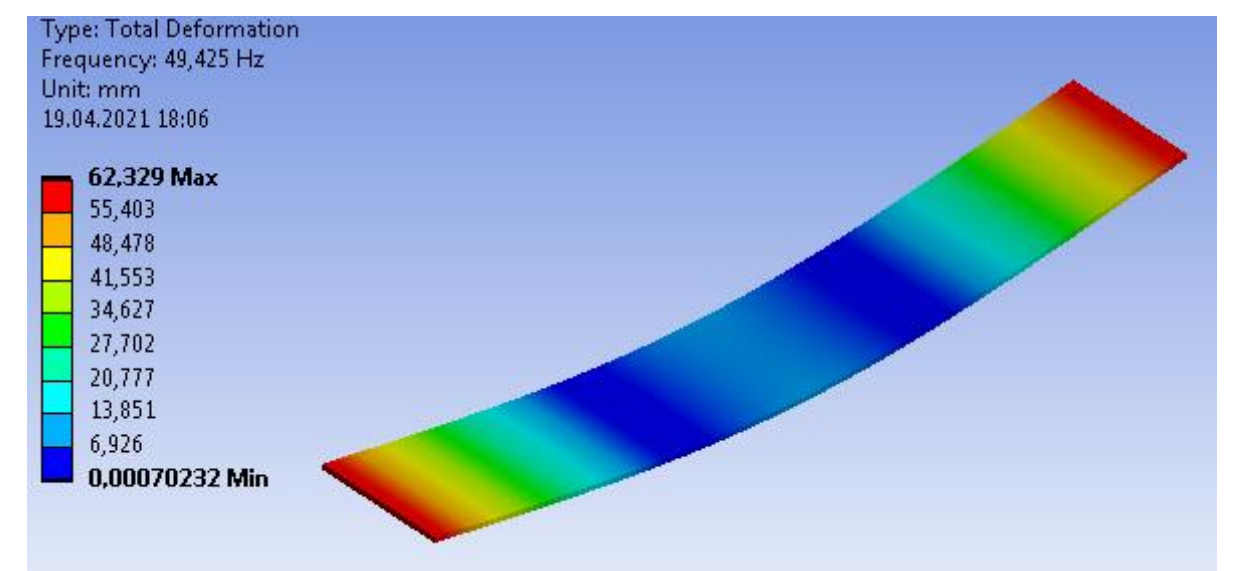

Fig. 6 The first natural frequency of the plate in the Ansys software (isometric view)

The isometric view (Fig. 6) shows the shape of the plate deformation. Also in Fig. 6 is shown the value of the first natural frequency of oscillations of the plate, obtained by modelling by the finite element method in the Ansys. It is $v_{f r s}=49,425 \mathrm{~Hz}$. Therefore, due to numerical simulations in the Ansys software, it was found that the natural frequency of the rod oscillations $v=49,22 \mathrm{~Hz}$ defined in paragraph 3 is reliable. This allows us to use the proposed paragraph 3 method to calculate the natural frequency of the plates for this type of fastening.

Fig. 7 shows that the investigated plate bends in a specific shape in the longitudinal direction and in the transverse. The surface of the deflected plate has the shape of a hyperboloid. 


\section{Convexity of the ends}

\section{Convexity of the middle}

Fig. 7 The first natural frequency of the plate in the Ansys software (transverse deflection view)

To sum up, numerical simulations in the Ansys software product showed that the elastic plate bends to form a hyperboloid surface at its first natural frequency. It allows us to use the hyperboloid's general equation to calculate the plate's natural frequency with this type of fastening.

\section{Calculation of the natural frequency of the plate by the approximate Rayleigh-Ritz method using the general hyperboloid equation}

To calculate the natural frequency of the plate for this type of fastening, we use the approximate Rayleigh-Ritz method. The deflection of the investigated plate, which has hinged supports at four points, occurs on the surface describing the hyperboloid (Fig. 7). Therefore, we can assume that the plate bent due to oscillations at the first natural frequency is part of the surface of the hyperboloid. For further calculation of the natural frequency of oscillations of the plate at this type of fastening, the scheme is composed (Fig. 8). 


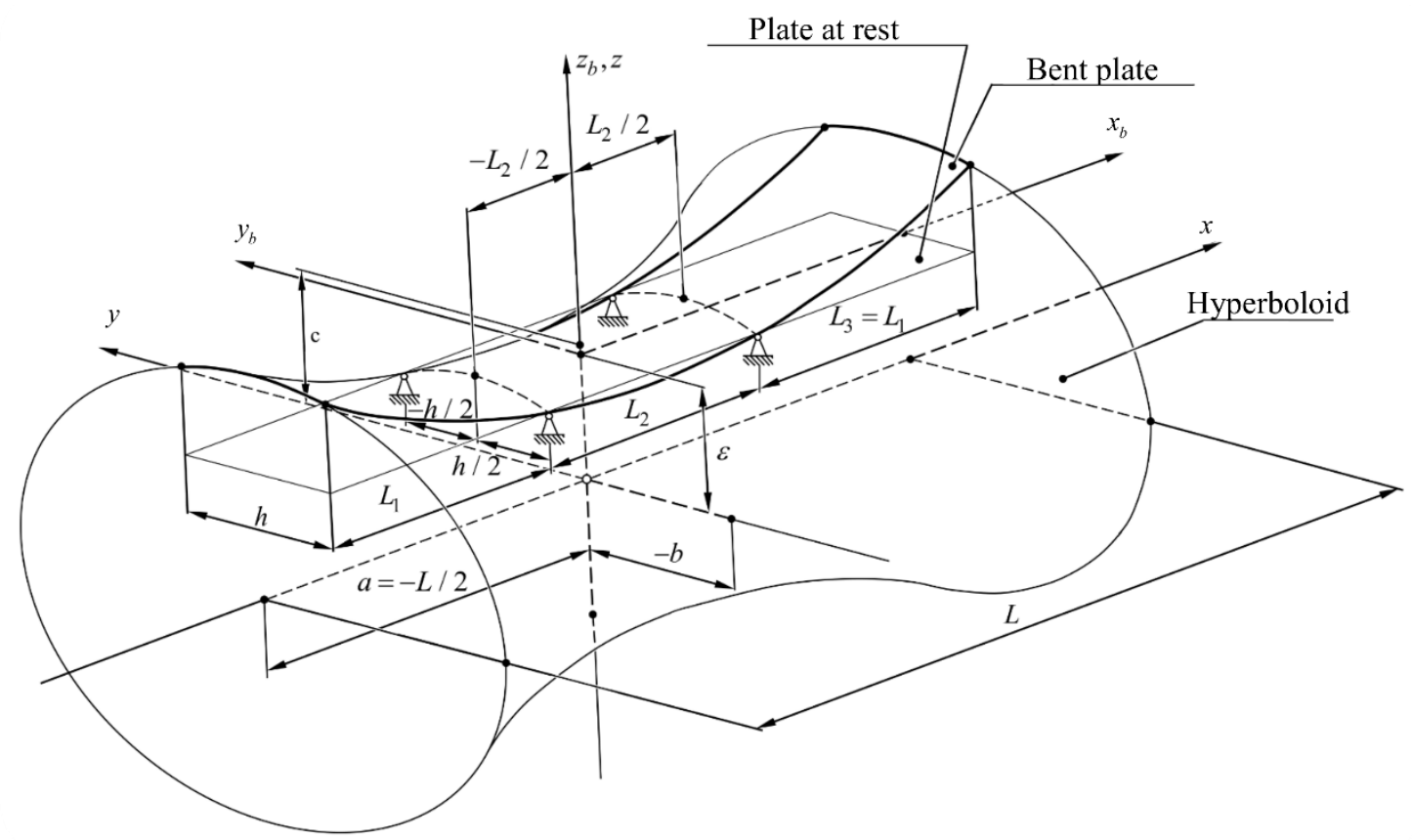

Fig. 8 Calculation scheme for determining the natural frequency of oscillations of the plate with the assumption that the oscillations occur on the hyperboloid surface, where $x, y, z$ - the coordinates of the absolute frame of reference; $x_{b}, y_{b}, z_{b}$-coordinates of the relative frame of reference; $\varepsilon$-displacement of the relative coordinate system along the axis $0 z$; $a, b, c$ - hyperboloid parameters

According to the calculation scheme in Fig. 8, the general equation of the hyperboloid will look like as:

$$
\frac{y^{2}}{b^{2}}+\frac{z^{2}}{c^{2}}-\frac{x^{2}}{a^{2}}=1
$$

To set the value $\varepsilon$, we use one of the four hinges. Then at the point with the coordinates $x=\frac{L_{2}}{2}, y=\frac{h}{2}$, the deflection along the axis $0 z$ will be equal to $\varepsilon$. Substituting these conditions into equation (25), we obtain:

$$
\frac{\left(\frac{h}{2}\right)^{2}}{b^{2}}+\frac{\varepsilon^{2}}{c^{2}}-\frac{\left(\frac{L_{2}}{2}\right)^{2}}{a^{2}}=1 .
$$

Entering the ratio of the sides $k=\frac{b}{c}$ and denoting $a=\frac{L}{2}$, we obtain the equation:

$$
\varepsilon=\sqrt{\left(\frac{b}{k}\right)^{2} \cdot\left[1+\frac{\left(\frac{L_{2}}{2}\right)^{2}}{\left(\frac{L}{2}\right)^{2}}-\frac{\left(\frac{h}{2}\right)^{2}}{b^{2}}\right]} .
$$


It should be noted that the value of the coefficient $k$ does not affect the value of the first natural frequency of the plate. Therefore, for further calculation, we take the value $k=1$. We also accept $b=0,6$.

As can be seen from Fig. 8, the deflection of the plate along the axis at any point will be equal:

$$
W(x, y)=z-\varepsilon .
$$

Using (26) - (28), the plate surface deflection equation will be of the form:

$$
W(x, y)=\sqrt{\left(\frac{b}{k}\right)^{2} \cdot\left[1+\frac{x^{2}}{\left(\frac{L}{2}\right)^{2}}-\frac{y^{2}}{b^{2}}\right]}-\sqrt{\left(\frac{b}{k}\right)^{2} \cdot\left[1+\frac{\left(\frac{L_{2}}{2}\right)^{2}}{\left(\frac{L}{2}\right)^{2}}-\frac{\left(\frac{h}{2}\right)^{2}}{b^{2}}\right]} .
$$

According to the Rayleigh-Ritz principle, the kinetic energy of oscillations of the plate is equal to the potential, i.e.:

$$
K=P .
$$

The ratio of kinetic energy $K$ to the square of the circular frequency of the plate $\omega^{2}$ at the deflection of the plate on the hyperboloid surface is determined by the expression [21]:

$$
\tilde{K}=\frac{K}{\omega^{2}}=\frac{1}{2} \int_{-L / 2}^{L / 2} \int_{-h / 2}^{h / 2} \rho b_{n} W(x, y)^{2} \mathrm{~d} x \mathrm{~d} y=5,61 \cdot 10^{-3} J \cdot s^{2} .
$$

The potential deflection energy at the first natural frequency of the plate oscillations on the hyperboloid surface will be determined by the following dependence [21]:

$$
P=\frac{1}{2} \int_{-L / 2}^{L / 2} \int_{-h / 2}^{h / 2} D\left(\begin{array}{c}
\left(\frac{\mathrm{d}^{2} W(x, y)}{\mathrm{d} x^{2}}+\frac{\mathrm{d}^{2} W(x, y)}{\mathrm{d} y^{2}}\right)^{2}+ \\
\left.+2(1-\mu)\left(\left(\frac{\mathrm{d}^{2} W(x, y)}{\mathrm{d} x \mathrm{~d} y}\right)^{2}-\frac{\mathrm{d}^{2} W(x, y)}{\mathrm{d} x^{2}} \cdot \frac{\mathrm{d}^{2} W(x, y)}{\mathrm{d} y^{2}}\right)\right)
\end{array}\right) \mathrm{d} x \mathrm{~d} y=550,14 J,
$$

where the multiplier $D$ can be found from the expression

$$
D=E \cdot b_{n}{ }^{3} /\left(12\left(1-\mu^{2}\right)\right) \text {, }
$$

$\mu=0,26-$ Poisson's ratio.

Finding the dependencies for determining the ratio of kinetic energy $K$ to the square of the circular frequency of the plate $\omega^{2}$ and the potential energy $P$ of the deflection of the plate on the hyperboloid surface according to (31) and (32), using expression (30) we obtain the value of its first natural frequency:

$$
v=\frac{\omega}{2 \pi}=\frac{1}{2 \pi} \cdot \sqrt{\frac{P}{\tilde{K}}}=\frac{1}{2 \pi} \cdot \sqrt{\frac{550,14}{5,61 \cdot 10^{-3}}}=49,8 \mathrm{~Hz} .
$$

The value of the natural frequency of the plate oscillations found by this method satisfies the required accuracy of calculations. Note that in contrast to the calculation of the natural frequency of the plate using Krylov-Duncan functions, this technique is less time-consuming. 


\section{Results of the experimental study.}

This paragraph describes an experimental study of the natural frequency of the plate vibration of our proposed interresonant vibrating machine with an electromagnetic drive. Fig. 10 shows an experimental sample of an interresonant vibrating table during operation at the first natural frequency of the plate.

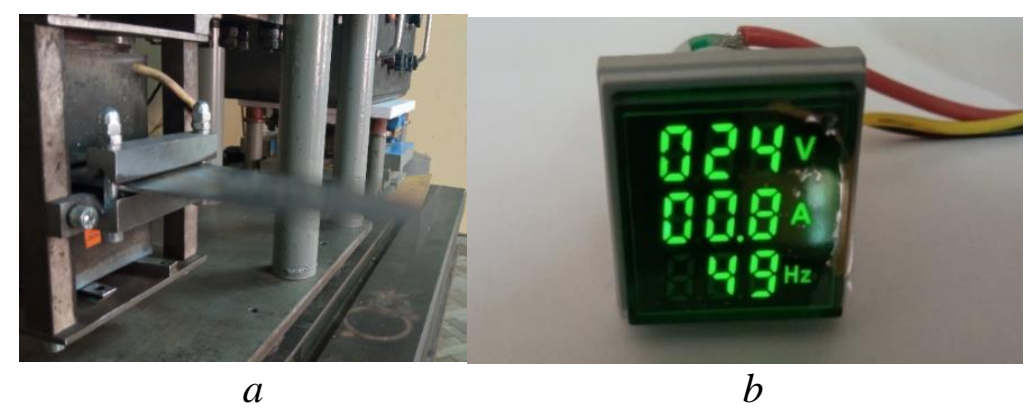

Fig. 10. Experimental sample of the three-mass inter-resonance vibrating table. Plate oscillations during resonance (a) and frequency sensor readings (b)

It was investigated that the first natural frequency of the rod is $\omega_{\text {exp }}=49 \mathrm{~Hz}$ (Fig. 10, b) and corresponds to the results of the calculations above using a frequency sensor.

\section{CONCLUSION}

1. The Krylov-Duncan functions have been selected for the type of plate attachment suggested by us, according to which the value of the first natural frequency of the plate oscillations is $v=49,22 \mathrm{~Hz}$. Using the Ansys simulation program, the value of the first natural frequency of the plate is $v_{f r s}=49,425 \mathrm{~Hz}$.

2. At the first natural frequency of oscillations, the elastic plate, bending, forms a hyperboloid surface. Therefore, the results obtained during the simulation allowed us to propose another technique based on the Rayleigh-Ritz method. The general hyperboloid equation is used as an essential function. It simplifies the calculation of the natural oscillation frequency of the plate for this type of fastening. Using this technique, the value of the first natural frequency of the plate is $49,8 \mathrm{~Hz}$.

3. The research on the experimental model of the inter-resonance vibrating table with the electromagnetic drive allowed us to generalize the results of numerical calculations and simulation modelling in the Ansys software. The obtained data confirmed the adequacy of the proposed calculation methods.

\section{REFERENCES}

[1] Ruchynskyi, M., Nazarenko, M., Pereginets, I., Kobylianskyi, O., Kisała, P., Abenov, A., Amirgaliyeva, Z. "Simulation and development of energy-efficient vibration machines operating in resonant modes“", Przegląd Elektrotechniczny 1(4), pp. 62 - 66, 2019. DOI: 10.15199/48.2019.04.11

[2] Lanets, O., Maistruk, P., Borovets, V., Derevenko, I. “Adjustment of parameters of three - mass interresonant vibrating machines with an inertial exciters", Industrial Process Automation in Engineering and Instrumentation 53, pp. 13 - 22, 2019. DOI: 10.23939/ istcipa2019.53.013 
[3] Lanets, O., Kachur, O. "Identification of ways to further improve of highly effective interresonance oscillatory systems", Industrial Process Automation in Engineering and Instrumentation 51, pp. $62-65,2017$.

[4] Lanets, O., Kachur, O., Borovets, V., Dmyterko, P., Derevenko, I., Zvarich, A. "Establishment of the original frequency of the continual section of the interreson research machine Rayleigh-Ritz method“", Industrial Process Automation in Engineering and Instrumentation 54, pp. 5 - 15, 2020. DOI: 10.23939/istcipa2020.54.005

[5] Chikh, A. "Free vibration analysis of simply supported P-FGM nanoplate using a nonlocal four variables shear deformation plate theory“, Strojnícky časopis - Journal of Mechanical Engineering 69(4), pp. 9 - 24, 2019. DOI: 10.2478/scjme-2019-0039

[6] Murin, J., Aminbaghai, M., Hrabovsky, J. "Elastostatic Analysis of the Spatial FGM Structures“, Strojnícky časopis - Journal of Mechanical Engineering 65(1), pp. 27 - 56, 2015. DOI: $10.1515 /$ scjme-2016-0003

[7] Taehyun, K., Usik, L. "Vibration Analysis of Thin Plate Structures Subjected to a Moving Force Using Frequency-Domain Spectral Element Method", Shock and Vibration Vol. 2018, pp. 1 - 27, 2018. DOI: 10.1155/2018/1908508

[8] Sharma, A. K., Sharma, P., Chauhan, P. S., Bhadoria, S. S. "Study on Harmonic Analysis of Functionally Graded Plates Using Fem“, International Journal of Applied Mechanics and Engineering 23 (4), pp. 941 - 961, 2018. DOI: 10.2478/ijame-2018-0053

[9] Zhao, X., Lee, Y. Y., Liew, K. M. "Free vibration analysis of functionally graded plates using the element-free kp-Ritz method", Journal of Sound and Vibration 319 (3-5), pp. 918 - 939, 2009. DOI: $10.1016 /$ j.jsv.2008.06.025

[10] Saeed, A., Hassan, H., Wael E. "Vibration attenuation using functionally graded material", World Academy of Science, Engineering and Technology 7(6), pp. 1111 1120, 2013. DOI: 10.5281 /zenodo. 1057221

[11] Reddy, J. N. "Theory and Analysis of Elastic Plates and Shells", 2-nd ed., CRC Press, Boca Raton, USA, 2007. ISBN 9780849384158, DOI: 10.1201/9780849384165

[12] Timoshenko S. P., Woinowsky-Krieger S. "Theory of Plates and Shells", 2-nd ed., McGraw-Hill, New York, USA, 1959. ISBN 0-07-064779-8

[13] Bochkarev, A. "Influence of boundary conditions on stiffness properties of a rectangular nanoplate“, Procedia Structural Integrity 6, pp. $174-181,2017$. DOI: 10.1016/j.prostr.2017.11.027

[14] Mahi, A., Adda Bedia, E.A., Tounsi, A. "A new hyperbolic shear deformation theory for bending and free vibration analysis of isotropic, functionally graded, sandwich and laminated composite plates“, Applied Mathematical Modelling 39 (9), pp. 2489 - 2508, 2015. DOI: $10.1016 /$ j.apm.2014.10.045

[15] Vescovini, R., Dozio, L., D'Ottavio, M., Polit, O. “On the application of the Ritz method to free vibration and buckling analysis of highly anisotropic plates", Composite Structures 192, pp. 460 - 474, 2018. DOI: 10.1016/j.compstruct.2018.03.017

[16] Kozbur H., Shkodzinsky O., Kozbur I., Gashchyn N. "Prediction of the boundary states for thin-walled axisymmetric shells under internal pressure and tension loads", Strojnícky časopis - Journal of Mechanical Engineering 70(1), pp. 57 - 68, 2020. DOI: 10.2478/scjme-2020-0006 
[17] Arvin, H. "The Flapwise Bending Free Vibration Analysis of Micro-rotating Timoshenko Beams Using the Differential Transform Method", Journal of Vibration and Control 24 (20), pp. 4868 - 4884, 2018. DOI: 10.1177/1077546317736706

[18] Chen, Y., Zhang, J., Zhang, H. "Free vibration analysis of rotating tapered Timoshenko beams via variational iteration method", Journal of Vibration and Control 23 (2), pp. 220 - 234, 2017. DOI: $10.1177 / 1077546315576431$

[19] Konieczny, M., Achtelik, H., Gasiak, G. "Research of maximum stress zones in circular plates with loades concentrated force“" Strojnícky časopis - Journal of Mechanical Engineering 70 (2), pp. 77 - 90, 2020. DOI: 10.2478/scjme-2020-0022

[20] Hlavaty, M., Starek, L., Musil, M., Hučko B. "Ultrasonic Defect Detection of Structural Plates Using Quasi-Rayleigh Waves“, Strojnícky časopis - Journal of Mechanical Engineering 67 (2), pp. 37 - 50, 2017. DOI: 10.1515/scjme-2017-0016

[21] Ponomarev, S. "Strength calculations in mechanical engineering", 3-rd ed., Mashgiz, Moscow, USSR, 1959. 